\title{
Asymptotically Tight Performance Bounds for Selection Diversity Over Beaulieu-Xie Fading Channels With Arbitrary Correlation
}

\author{
Adebola Olutayo, Student Member, IEEE, Julian Cheng, Senior Member, IEEE, \\ Jonathan Holzman, Member, IEEE
}

\begin{abstract}
A new (Beaulieu-Xie) fading model was recently proposed to describe line-of-sight and non-line-of-sight components in wireless channels. In this work, we consider both outage probability and error rate performance of selective combining over this new fading channel model with arbitrary channel correlation. Closed-form expressions are obtained for asymptotically tight upper and lower bounds. The analytical results are verified by Monte Carlo simulations.
\end{abstract}

\section{INTRODUCTION}

A fading model of particular practical importance is the Ricean fading model. The Ricean fading model benefits from its ability to characterize wireless systems with a line-of-sight (LOS) component. Such a condition often manifests itself in wireless systems when the received LOS signal dominates over the received multi-path (non-LOS) signals. The Ricean fading model has limitations, however, in that it does not have a flexible fading parameter and its diversity order is only one. This restricts its ability to characterize wireless channels and mitigate fading over a broad range of conditions [1].

The Nakagami- $m$ fading model has emerged from an effort to characterize wireless channels over a broad range of conditions. This is done by via fading parameter $m$. It has proven to be a highly effective model for wireless systems with multipath signals, but it has also been shown to have faults for wireless systems with both LOS and multi-path signals [2]-[4]. Because of this, the Nakagami- $m$ fading model is considered to be valid for wireless systems operating with only multi-path signals.

An alternative fading model has recently been proposed by Beaulieu and Xie [1] in an effort to overcome the challenges of the Ricean and Nakagami- $m$ fading models. This new fading model, called the Beaulieu-Xie fading model in this work, is derived from a non-central Chi-distribution. Such a formulation allows it to characterize wireless channels over a broad range of conditions, by way of its fading parameter, in wireless systems with both LOS and multi-path signals. This work analyzes the performance of this new fading model.

To facilitate fair analyses for the new Beaulieu-Xie fading model, its prospects for diversity reception are characterized without an assumption of independent fading in the diversity channels. Correlated fading is considered in the analyses because the assumption of independence is only valid when there is sufficient physical spacing between channels. This is often not the case for large multiple-input-multiple-output implementations [5]. Thus, we consider correlation to quantify the performance degradation in the gain for selection combining (SC) [6], [7]. This work employs the techniques of [8], using a correlation matrix of the Gaussian random variable $(\mathrm{RV})$, to analyze the performance a diversity reception over correlated fading channels. To the authors' best knowledge, this paper represents the first work to study the performance of diversity reception for the Beaulieu-Xie fading model.

\section{System MODEL}

We consider diversity reception with $N$ branches adhering to the Beaulieu-Xie fading model. The received signal is

$$
\mathbf{y}=\mathbf{z} x+\mathbf{n}
$$

where $x$ is the transmitted signal, $\mathbf{n}$ is a random vector denoting additive Gaussian white noise (AWGN) whose variances are assumed to be normalized, and $\mathbf{z}$ is the fading channel vector, i.e., the real fading amplitude. In addition, $\mathbf{z}=\left[z_{1}, \ldots, z_{N}\right]^{T}=\left[\sqrt{\bar{\gamma}_{1}} Z_{1}, \ldots, \sqrt{\bar{\gamma}_{N}} Z_{N}\right]^{T}$, where [.] $]^{T}$ represents the transpose, $\bar{\gamma}_{n}$ is the average received signalto-noise ratio (SNR) of the $n$th branch, and $Z_{n}$ is the powernormalised fading amplitude.

The respective output SNRs for SC diversity reception is

$$
\gamma_{S C}=\max \left(z_{1}^{2} \cdots, z_{n}^{2}\right) .
$$

The Beaulieu-Xie RV associated with the $n$th branch is generated by

$$
Z_{n}=\sqrt{\sum_{i=1}^{2 m} X_{n, i}^{2}} ; \quad \forall n=1, \ldots N
$$

where $X_{n, i}, \forall i=1, \ldots 2 m$ are Gaussian RVs with mean $\mu$, variance $\left(\frac{1}{2 m}\right)$ and $m$ is a half-integer, representing the fading parameter that controls the shape of the probability density function (pdf) of the fading model. The $n$th component of $\mathbf{z}$ can be obtained by generating $N \times 2 m$ matrix of Gaussian RVs, $\mathbf{B}$, whose entries are $b_{n, i}$ whose $m$ th column is denoted as $\mathbf{b}_{m}$, such that $\mathbf{B}=\left(\mathbf{b}_{1}, \ldots, \mathbf{b}_{2 m}\right)$. We obtain the vector, $\mathbf{b}=$ 
$\left[\mathbf{b}_{1}^{T}, \ldots, \mathbf{b}_{2 m}^{T}\right]^{T}=\left[b_{1,1}, b_{2,1}, \ldots, b_{n, 2 m}\right]^{T}$. We can, therefore, express the fading amplitude over the $n$th branch as

$$
z_{n}=\sqrt{\sum_{i=1}^{2 m} b_{n, i}^{2}} ; \quad \forall n=1, \ldots N .
$$

The pdf of $\mathbf{b}$ is expressed as

$$
\begin{aligned}
f_{\mathbf{b}}(\mathbf{b}) & =\frac{1}{\sqrt{(2 \pi)^{2 m N}\left|\mathbf{R}_{\mathbf{b}}\right|}} \\
& \times \exp \left(-\frac{1}{2}\left(\mathbf{b}-\mu_{\mathbf{b}}\right)^{T} \mathbf{R}_{\mathbf{b}}^{-1}\left(\mathbf{b}-\mu_{\mathbf{b}}\right)\right)
\end{aligned}
$$

where $\boldsymbol{\mu}_{\boldsymbol{b}}$ is the $2 m N \times 1$ mean vector and $\mathbf{R}_{\mathbf{b}}$ is the $2 m N \times 2 m N$ covariance matrix of $\mathbf{b}$. The determinant of the covariance matrix $\mathbf{R}_{\mathbf{b}}$ is expressed in terms of the correlation matrix $\mathbf{C}_{\mathbf{b}}$ by [8]

$$
\left|\mathbf{R}_{\mathbf{b}}\right|=\frac{\left(\prod_{n=1}^{N} \bar{\gamma}_{n}^{2 m}\right)\left|\mathbf{C}_{\mathbf{b}}\right|}{(2 m)^{2 m N}} .
$$

Note that the diversity order $m N$ for this new fading model is the same as that of the Nakagami- $m$ fading model, for the same value of $m$, but the presence of LOS components makes the former an improved channel model [1].

\section{RELATIONSHIP BETWEEN THE POWER CORRELATION AND GaUsSian CORRELATION COEFFICIENT}

Using Cholesky decomposition, as shown in [6], we obtain the correlation coefficient between RVs $X_{n, i}$ and $X_{j, k}$ with mean $\mu$ and variance $\frac{1}{2 m}$. It can be shown that $X_{n, i}=$ $\rho_{(n, i)(j, k)} X_{j, k}+\sqrt{1-\rho_{(n, i)(j, k)}^{2}} W$, where $W$ is a Gaussian $\mathrm{RV}$ with zero mean and variance $\frac{1}{2 m}$ and it is independent of $X_{j, k}$. This leads to the relationship between the power correlation coefficient and correlation coefficient of the Gaussian $\mathrm{RV}$ s as

$$
\rho_{z_{n_{1}}^{2} z_{n_{2}}^{2}}=\frac{K-(2 m)^{2} T^{2}}{3 U^{2}-2 m T^{2}}
$$

where

$$
\begin{gathered}
K=\sum_{i=1}^{2 m} \sum_{k=1}^{2 m}\left(3 U^{2} \rho_{(n, i)(j, k)}^{2}+T^{2}\left(1-\rho_{(n, i)(j, k)}^{2}\right)\right), \\
T=\frac{1}{2 m}+\mu^{2}, \text { and } U=\frac{1}{2 m} .
\end{gathered}
$$

\section{Performance Analysis}

It is important to understand the effects of correlated fading on the performance of a diversity system. Outage probability (OP) and error rates are two metrics that can be used to quantify these effects. We analyze the performance of this fading model by computing the bounds of the OP and error rate according to [8]. Here, we set the SNR threshold, $\gamma_{t h}$ for OP, below which we measure the occurrence of outages [8].

\section{A. Bounds on the pdf}

We find the lower and upper bounds of the pdf of $\mathbf{b}$ by considering the exponential component of (4). A Rayleigh quotient is applied as $R_{y}(\mathbf{P}, \mathbf{x})=\frac{\mathbf{x}^{T} \mathbf{P} \mathbf{x}}{\mathbf{x}^{T} \mathbf{x}}$, which has a minimum value of $\lambda_{\min }$ (i.e., a small eigenvalue of $\mathbf{P}$ ) and maximum value of $\lambda_{\max }$ (i.e., a large eigenvalue of $\mathbf{P}$ ). Applying this to our case gives $\lambda_{\min } \leq \frac{\mathbf{b}^{T} \mathbf{R}_{\mathbf{b}}^{-1} \mathbf{b}}{\mathbf{b}^{T} \mathbf{b}} \leq \lambda_{\max }$, where $\mathbf{R}_{\mathbf{b}}$ is a positive definite matrix, such that $\lambda_{i} \geq 0$. Thus, we take $\lambda_{\min }=0$ to give

$$
0 \leq \mathbf{b}^{T} \mathbf{R}_{\mathbf{b}}^{-1} \mathbf{b} \leq \lambda_{\max } \mathbf{b}^{T} \mathbf{b} .
$$

We expand the exponential component with an assumption that $\mathbf{R}_{\mathbf{b}}^{-1^{T}}=\mathbf{R}_{\mathbf{b}}^{-1}$, and we note that $\mathbf{R}_{\mathbf{b}}^{-1}=\mathbf{R}_{\mathbf{b}}$ as $\mathbf{b} \rightarrow \mathbf{0}$. This gives

$$
\begin{aligned}
& \left(\mathbf{b}-\mu_{\mathbf{b}}\right)^{T} \mathbf{R}_{\mathbf{b}}^{-1}\left(\mathbf{b}-\mu_{\mathbf{b}}\right) \\
& =\mathbf{b}^{\mathbf{T}} \mathbf{R}_{\mathbf{b}}^{-1} \mathbf{b}+\mu_{\mathbf{b}}{ }^{T} \mathbf{R}_{\mathbf{b}}^{-1} \mu_{\mathbf{b}}+2\left|\mu_{\mathbf{b}}{ }^{T} \mathbf{R}_{\mathbf{b}}^{-1} \mathbf{b}\right|
\end{aligned}
$$

Employing the 2-norm of the matrix as $\|\mathbf{b}\|=\sqrt{\mathbf{b}^{\mathbf{T}} \mathbf{b}}=$ $\sqrt{r}$, where $r$ is the radius of the $2 m N$-dimensional sphere, eq. (8) becomes

$$
\begin{aligned}
& -2\left\|\mu_{\mathbf{b}}{ }^{T} \mathbf{R}_{\mathbf{b}}^{-1}\right\| \sqrt{r} \leq\left(\mathbf{b}-\mu_{\mathbf{b}}\right)^{T} \mathbf{R}_{\mathbf{b}}^{-1}\left(\mathbf{b}-\mu_{\mathbf{b}}\right) \\
& \leq \lambda_{\max } r+2\left\|\mu_{\mathbf{b}}{ }^{T} \mathbf{R}_{\mathbf{b}}^{-1}\right\| \sqrt{r} .
\end{aligned}
$$

The upper and lower bounds of the pdf can then be obtained as

$$
\begin{aligned}
& f_{\mathbf{b}}(\mathbf{0}) \exp \left(-\frac{1}{2}\left(\lambda_{\max } r+2\left\|\mu_{\mathbf{b}}{ }^{T} \mathbf{R}_{\mathbf{b}}^{-1}\right\| \sqrt{r}\right)\right) \leq f_{\mathbf{b}}(\mathbf{b}) \\
& \leq f_{\mathbf{b}}(\mathbf{0}) \exp \left(\left\|\mu_{\mathbf{b}}{ }^{T} \mathbf{R}_{\mathbf{b}}^{-1}\right\| \sqrt{r}\right) .
\end{aligned}
$$

\section{B. Outage Probability Bounds on SC}

We express the outage probability for SC with respect to a signal threshold, $\gamma_{t h}$, as

$$
P_{\text {out }}^{S C}\left(\gamma_{t h}\right)=\operatorname{Pr}\left\{\gamma_{S C} \leq \gamma_{t h}\right\}
$$

Substituting (3) into (2) gives

$$
\begin{aligned}
P_{\text {out }}^{S C}\left(\gamma_{t h}\right) & =\operatorname{Pr}\left\{\max _{n}\left\{\sum_{i=1}^{2 m} b_{n, i}^{2}\right\} \leq \gamma_{t h}\right\} \\
& =\int_{\gamma_{S C} \leq \gamma_{t h}} f_{\mathbf{b}}(\mathbf{b}) d \mathbf{b} .
\end{aligned}
$$

1) Asymptotic Outage Probability Approximation of SC: The asymptotic outage probability approximation is obtained by substituting $f_{\mathbf{b}}(\mathbf{b}) \approx f_{\mathbf{b}}(\mathbf{0})$ into (12) and simplifying to give

$$
P_{\text {out }, \infty}^{S C}\left(\gamma_{t h}\right)=f_{\mathbf{b}}(\mathbf{0}) \prod_{n=1}^{N} \int d \mathbf{b}_{n}
$$


where $d \mathbf{b}_{n}=\Pi_{n=1}^{N} d b_{n, i}$. The integral in (13) is the volume of a $2 m$-dimensional sphere with radius, $r=\sqrt{\gamma_{t h}}$, and ultimately, eq. (13) can be expressed as

$$
P_{\text {out }, \infty}^{S C}\left(\gamma_{t h}\right)=f_{\mathbf{b}}(\mathbf{0})\left[\frac{\left(2 \sqrt{\gamma_{t h}}\right)^{(2 m+1)} \pi^{m} \Gamma(m+1)}{(2 m+1) !}\right]^{N} \text {. }
$$

2) Lower Bound Outage Probability of SC: The lower bound of the outage probability is obtained by replacing $f_{\mathbf{b}}(\mathbf{0})$ with the lower bound of the pdf obtained in (10). Substituting the result into (13) gives

$$
P_{\text {out }, L B}^{S C}\left(\gamma_{t h}\right)=O_{L B} \int_{\gamma_{S C} \leq \gamma_{t h}} d \mathbf{b}
$$

where

$$
\begin{aligned}
O_{L B} & =f_{\mathbf{b}}(0) \exp \left(-\frac{1}{2}\left(\lambda_{\max } N \gamma_{t h}\right.\right. \\
& \left.\left.+2\left\|\mu_{\mathbf{b}}{ }^{T} \mathbf{R}_{\mathbf{b}}^{-1}\right\| \sqrt{N \gamma_{t h}}\right)\right)
\end{aligned}
$$

Equation (15) can be further simplified to

$$
P_{\text {out }, L B}^{S C}\left(\gamma_{t h}\right)=O_{L B}\left[\frac{\left(2 \sqrt{\gamma_{t h}}\right)^{(2 m+1)} \pi^{m} \Gamma(m+1)}{(2 m+1) !}\right]^{N}
$$

3) Upper Bound Outage Probability of SC: The upper bound of the outage probability is obtained by replacing $f_{\mathbf{b}}(\mathbf{0})$ with the upper bound of the pdf obtained in (10), and by substituting the result into (13). This gives

$$
P_{\text {out }, U B}^{S C}\left(\gamma_{t h}\right)=O_{U B} \int_{\gamma_{S C} \leq \gamma_{t h}} d \mathbf{b}
$$

where $O_{U B}=f_{\mathbf{b}}(\mathbf{0}) \exp \left(\left\|\mu_{\mathbf{b}}^{T} \mathbf{R}_{\mathbf{b}}^{-1}\right\| \sqrt{N \gamma_{t h}}\right)$. Equation (17) is further simplified to

$$
P_{\text {out }, U B}^{S C}\left(\gamma_{t h}\right)=O_{U B}\left[\frac{\left(2 \sqrt{\gamma_{t h}}\right)^{(2 m+1)} \pi^{m} \Gamma(m+1)}{(2 m+1) !}\right]^{N}
$$

\section{Error Rate Bounds on SC}

We can express the error rate for $\mathrm{SC}$ as

$$
\begin{aligned}
P_{e}^{S C} & =E\left[p Q\left(\sqrt{q \max _{n}\left\{\sum_{i=1}^{2 m} b_{n, i}^{2}\right\}}\right)\right] \\
& =p \int_{-\infty}^{\infty} Q\left(\sqrt{q \max _{n}\left\{\sum_{i=1}^{2 m} b_{n, i}^{2}\right\}}\right) f_{\mathbf{b}}(\mathbf{b}) d \mathbf{b}
\end{aligned}
$$

where $E[\cdot]$ denotes the expectation operation, and the Gaussian $Q$-function is $Q(x)=\frac{1}{\sqrt{2 \pi}} \int_{x}^{\infty} \exp \left(\frac{-t^{2}}{2}\right) d t$. For the special case of binary phase shift keying (BPSK), we have, $p=1$ and $q=2$.
1) Asymptotic Error Rate Approximation of SC: The asymptotic error rate approximation is obtained by substituting $f_{\mathbf{b}}(\mathbf{b}) \approx f_{\mathbf{b}}(\mathbf{0})$ into (19) to give

$$
P_{e}^{S C}=p f_{\mathbf{b}}(\mathbf{0}) \int_{-\infty}^{\infty} Q\left(\sqrt{q \max _{n}\left\{\sum_{i=1}^{2 m} b_{n, i}^{2}\right\}}\right) d \mathbf{b}
$$

which can be simplified to [8]

$$
P_{e, \infty}^{S C}=p f_{\mathbf{b}}(\mathbf{0}) \frac{2^{m N-1} \pi^{m N} \Gamma(m N+1 / 2)}{q^{m N} \sqrt{\pi} \Gamma^{N}(m+1)} .
$$

2) Lower Error Rate Bound of SC: The lower bound of the error rate is obtained by changing the integral region in (19) according to

$$
P_{e}^{S C}=p \int_{R_{S C} \leq R_{t h}^{2}} Q\left(\sqrt{q \max _{n}\left\{\sum_{i=1}^{2 m} b_{n, i}^{2}\right\}}\right) f_{\mathbf{b}}(\mathbf{b}) d \mathbf{b}
$$

where $R_{S C}=\max _{n}\left\{\sum_{i=1}^{2 m} b_{n, i}^{2}\right\}$. Substituting $f_{\mathbf{b}}(\mathbf{b})$ with the lower bound of the pdf in (10) into (22), where $r=R_{t h}$, and after some simplifications, we have

$$
P_{e, L B}^{S C}=p \times E_{L B} \times V\left(R_{t h}\right)
$$

where

$$
\begin{aligned}
E_{L B} & =f_{\mathbf{b}}(\mathbf{0}) \exp \left(-\frac{1}{2}\left(\lambda_{\max } N R_{t h}^{2}\right.\right. \\
& \left.\left.+2\left\|\mu_{\mathbf{b}}{ }^{T} \mathbf{R}_{\mathbf{b}}^{-1}\right\| \sqrt{N} R_{t h}\right)\right)
\end{aligned}
$$

and

$$
\begin{aligned}
V\left(R_{t h}\right) & =\frac{\pi^{m N}}{\Gamma^{N}(m+1)}\left(R_{t h}^{2 m N} Q\left(\sqrt{q} R_{t h}\right)\right. \\
& \left.+\frac{2^{m N-1}}{q^{m N} \sqrt{\pi}} \gamma\left(\frac{2 m N+1}{2}, \frac{q}{2} R_{t h}^{2}\right)\right)
\end{aligned}
$$

where $\gamma(\cdot, \cdot)$ is the incomplete gamma function as, $\gamma(\alpha, x)=$ $\int_{0}^{x} e^{-t} t^{\alpha-1} d t[8]$.

3) Upper Error Rate Bound of SC: The upper error rate bound is obtained by splitting the integral region according to

$$
\begin{aligned}
& P_{e, U B}^{S C}=p \int_{R_{S C} \leq R_{t h}^{2}} Q\left(\sqrt{q \max _{n}\left\{\sum_{i=1}^{2 m} b_{n, i}^{2}\right\}}\right) f_{\mathbf{b}}(\mathbf{b}) d \mathbf{b} \\
& +p \int_{R_{S C}>R_{t h}^{2}} Q\left(\sqrt{\left.q \max _{n}\left\{\sum_{i=1}^{2 m} b_{n, i}^{2}\right\}\right)} f_{\mathbf{b}}(\mathbf{b}) d \mathbf{b} .\right.
\end{aligned}
$$


We consider the upper bound of the pdf obtained in (10) such that (24) can be expressed as

$$
\begin{aligned}
& P_{e, U B}^{S C}=p \times E_{U B} \int_{R_{S C} \leq R_{t h}^{2}} Q\left(\sqrt{q \max _{n}\left\{\sum_{i=1}^{2 m} b_{n, i}^{2}\right\}}\right) d \mathbf{b} \\
& +p f_{\mathbf{b}}\left(\mu_{\mathbf{b}}\right) \int_{R_{S C}>R_{t h}^{2}} Q\left(\sqrt{q \max _{n}\left\{\sum_{i=1}^{2 m} b_{n, i}^{2}\right\}}\right) d \mathbf{b}
\end{aligned}
$$

where the first integral is simplified as in (23) and $E_{U B}=$ $f_{\mathbf{b}}(\mathbf{0}) \exp \left(\left\|\mu_{\mathbf{b}}{ }^{T} \mathbf{R}_{\mathbf{b}}^{-1}\right\| \sqrt{N} R_{t h}\right)$. The second integral is obtained as

$$
\begin{aligned}
& \int_{R_{S C}>R_{t h}^{2}} Q\left(\sqrt{q \max _{n}\left\{\sum_{i=1}^{2 m} b_{n, i}^{2}\right\}}\right) d \mathbf{b}=G\left(R_{t h}\right) \\
= & {\left[\frac{\pi^{m N} 2^{m N-1}}{q^{m N} \sqrt{\pi}} \frac{\Gamma\left(m N+\frac{1}{2}\right)}{\Gamma^{N}(m+1)}-\frac{\pi^{m N}}{\Gamma^{N}(m+1)}\left(R_{t h}^{2 m N}\right.\right.} \\
\times & \left.\left.Q\left(\sqrt{q} R_{t h}\right)+\frac{2^{m N-1}}{q^{m N} \sqrt{\pi}} \gamma\left(\frac{2 m N+1}{2}, \frac{q}{2} R_{t h}^{2}\right)\right)\right] .
\end{aligned}
$$

The upper error rate bound for SC is obtained by substituting $V\left(R_{t h}\right)$ and (26) into (25).

\section{DisCUSSION ON THE TIGHTNESS OF BOUNDS}

\section{A. Tightness of the Outage Probability Bound}

We consider the exponential component of the lower and upper bounds of the outage probability for SC in (15) and (17), respectively. A close look at the lower bound expression shows that it differs from the asymptotic and upper bound by the exponential component. Also, a close look at the upper bound expression shows that it differs from the asymptotic and lower bound by the exponential component. We also note that an increase to the average SNR per branch by a factor $K$ results in an increase in the covariance matrix $\mathbf{R}_{\mathbf{b}}$ and eventually a decrease in $\mathbf{R}_{\mathrm{b}}^{-1}$ by the same factor. This leads to a decrease in $\lambda_{\max }$ (the largest eigenvalue of $\mathbf{R}_{\mathbf{b}}^{-1}$ ) by that same factor (i.e., $\frac{\lambda_{\max }}{K}$ ). Thus, we can state that

$$
\begin{aligned}
& \lim _{K \rightarrow \infty}=\frac{P_{o p, L B}}{P_{o p, U B}} \\
& =\frac{\exp \left(-\frac{1}{2}\left(\frac{\lambda_{\max } \gamma_{t h}}{K}+2\left\|\frac{\mu_{\mathbf{b}}{ }^{T} \mathbf{R}_{\mathbf{b}}^{-1}}{K}\right\| \sqrt{\gamma_{t h}}\right)\right)}{\exp \left(\left\|\frac{\mu_{\mathbf{b}} \mathbf{R}_{\mathbf{b}}^{-1}}{K}\right\| \sqrt{\gamma_{t h}}\right)}=1 .
\end{aligned}
$$

Note that the bounds of the outage probability are seen to get tighter as the SNR increases. This effect is seen in the upcoming outage probability results of this work.

\section{B. Tightness of the Error Rate Bound}

Given the definition of the incomplete gamma funtion as

$$
\Gamma(s)=\lim _{x \rightarrow \infty} \gamma(s, x)
$$

and the Chernoff bound of the $Q$-function as

$$
Q(x) \leq e^{-\frac{x^{2}}{2}}, \quad x>0
$$

we apply these definitions to $V$ and $G$, and then (22) and (25), to show the tightness of the error rate bounds, according to

$$
\begin{gathered}
\lim _{R_{t h} \rightarrow \infty} V=\frac{2^{m N-1}}{\sqrt{\pi} q^{m N}} \Gamma\left(m N+\frac{1}{2}\right) \\
\lim _{R_{t h} \rightarrow \infty} G=0 .
\end{gathered}
$$

We then consider the exponential component, where we see that an increase in the average SNR per branch by a factor $K$ results in an increase in the covariance matrix $\mathbf{R}_{\mathbf{b}}$. This leads to a decrease in $\mathbf{R}_{\mathbf{b}}^{-1}$ and eventually a decrease in $\lambda_{\max }$ by the same factor $K$. Thus, we find the limit to be

$$
\begin{aligned}
& \lim _{K \rightarrow \infty}=\frac{P_{e, L B}}{P_{e, U B}} \\
& =\frac{\exp \left(-\frac{1}{2}\left(\frac{\lambda_{\max } R_{t h}^{2}}{K}+2\left\|\mu_{\mathbf{b}}{ }^{T} \mathbf{R}_{\mathbf{b}}^{-1}\right\| R_{t h}\right)\right)}{\exp \left(\left\|\mu_{\mathbf{b}}{ }^{T} \mathbf{R}_{\mathbf{b}}^{-1}\right\| R_{t h}\right)} .
\end{aligned}
$$

Expressing $R_{t h}$ in terms of $K$, for instance $R_{t h}=\sqrt{K}$, gives

$$
\begin{aligned}
& \lim _{K \rightarrow \infty}=\frac{P_{e, L B}}{P_{e, U B}} \\
& =\frac{\exp \left(-\frac{1}{2}\left(\frac{\lambda_{\max } \gamma_{t h}}{K}+2\left\|\frac{\mu_{\mathbf{b}}{ }^{T} \mathbf{R}_{\mathbf{b}}^{-1}}{K}\right\| \sqrt{K}\right)\right)}{\exp \left(\left\|\frac{\mu_{\mathbf{b}}{ }^{T} \mathbf{R}_{\mathbf{b}}^{-1}}{K}\right\| \sqrt{K}\right)}=1 .
\end{aligned}
$$

It is seen that as $R_{t h}$ is increased, the lower and upper error rate bounds approach the asymptotic bounds.

\section{NumericAl RESUlts}

Figure 1 shows the outage probability curves of SC for a 2-branch diversity system over correlated Beaulieu-Xie fading channels with coherent BPSK modulation and a fading parameter of $m=1.5$. Correlation structure of the form $\mathbf{C}_{\mathbf{b}}=\left[\mathbf{C}_{1}, \mathbf{0}_{2 \times 2}, \mathbf{0}_{2 \times 2} ; \mathbf{0}_{2 \times 2}, \mathbf{C}_{2}, \mathbf{0}_{2 \times 2} ; \mathbf{0}_{2 \times 2}, \mathbf{0}_{2 \times 2}, \mathbf{C}_{3}\right]$, where $\mathbf{C}_{1}=\mathbf{C}_{2}=\mathbf{C}_{3}=[1, \sqrt{0.35} ; \sqrt{0.35}, 1]$ is considered. The lower and upper bounds converge to the asymptotic bound at high SNR.

Figure 2 shows a comparison of outage probability curves of SC for a 2-branch diversity system over correlated and independent Beaulieu-Xie fading channel$\mathrm{s}$ with coherent BPSK modulation and a fading parameter of $m=1.5$. Correlation structure of the for$\mathrm{m}_{\mathbf{b}}=\left[\mathbf{C}_{1}, \mathbf{0}_{2 \times 2}, \mathbf{0}_{2 \times 2} ; \mathbf{0}_{2 \times 2}, \mathbf{C}_{2}, \mathbf{0}_{2 \times 2} ; \mathbf{0}_{2 \times 2}, \mathbf{0}_{2 \times 2}, \mathbf{C}_{3}\right]$, where $\mathbf{C}_{1}=\mathbf{C}_{2}=\mathbf{C}_{3}=[1, \sqrt{0.8} ; \sqrt{0.8}, 1]$ and $\mathbf{C}_{1}=\mathbf{C}_{2}=$ $\mathbf{C}_{3}=[1,0 ; 0,1]$ are considered for correlation and independence, respectively. The independent channels outperform the correlated channels.

Figure 3 shows the BER plot of SC for a 2-branch diversity system over correlated Beaulieu-Xie fading channels with coherent BPSK modulation and a fading parameter of $m=1.5$. Correlation structure of the for$\mathrm{m} \mathbf{C}_{\mathbf{b}}=\left[\mathbf{C}_{1}, \mathbf{0}_{2 \times 2}, \mathbf{0}_{2 \times 2} ; \mathbf{0}_{2 \times 2}, \mathbf{C}_{2}, \mathbf{0}_{2 \times 2} ; \mathbf{0}_{2 \times 2}, \mathbf{0}_{2 \times 2}, \mathbf{C}_{3}\right]$, 


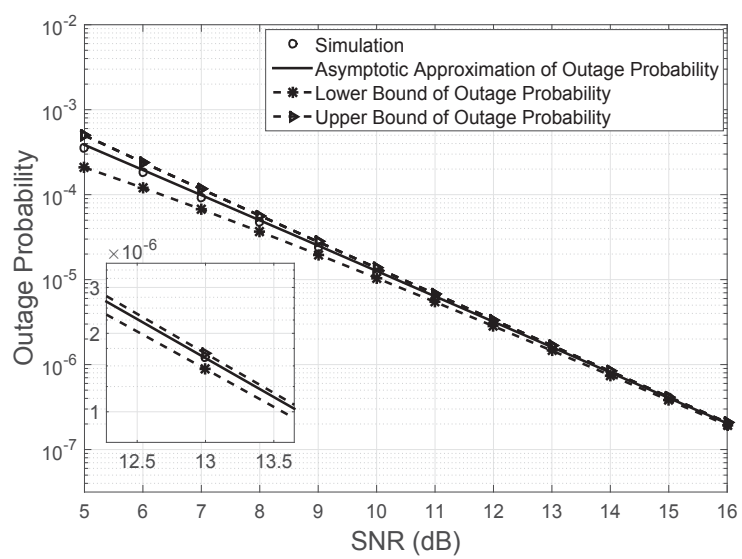

Fig. 1. Outage probability curves for the asymptotic approximation, lower bound, and upper bound for SC over correlated BeaulieuXie fading channels. The number of branches is $N=2$, the fading parameter is $m=1.5$, and the correlation structure is $\mathbf{C}_{\mathbf{b}}=$ $\left[\mathbf{C}_{1}, \mathbf{0}_{2 \times 2}, \mathbf{0}_{2 \times 2} ; \mathbf{0}_{2 \times 2}, \mathbf{C}_{2}, \mathbf{0}_{2 \times 2} ; \mathbf{0}_{2 \times 2}, \mathbf{0}_{2 \times 2}, \mathbf{C}_{3}\right]$, where $\mathbf{C}_{1}=\mathbf{C}_{2}=$ $\mathbf{C}_{3}=[1, \sqrt{0.35} ; \sqrt{0.35}, 1]$

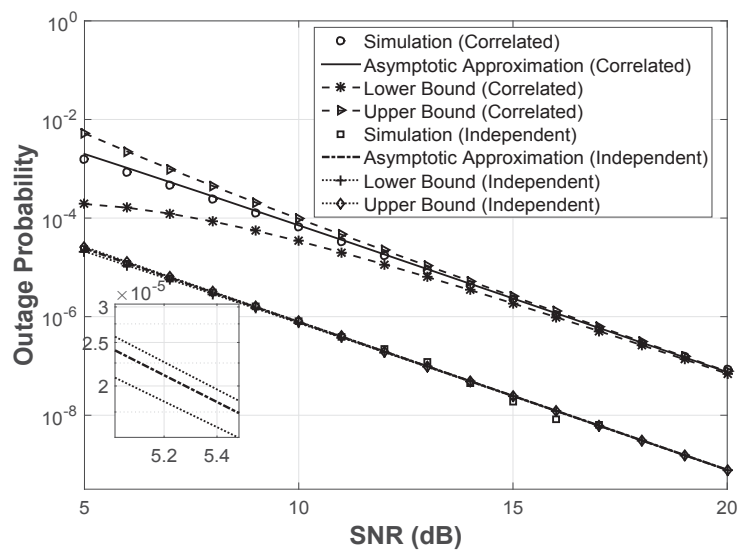

Fig. 2. Outage probability curves for the asymptotic approximation, lower bound, and upper bound for SC over correlated and independent Beaulieu-Xie fading channels. The number of branches is $N=2$, the fading parameter is $m=1.5$, and the correlation structure is $\mathbf{C}_{\mathbf{b}}=$ $\left[\mathbf{C}_{1}, \mathbf{0}_{2 \times 2}, \mathbf{0}_{2 \times 2} ; \mathbf{0}_{2 \times 2}, \mathbf{C}_{2}, \mathbf{0}_{2 \times 2} ; \mathbf{0}_{2 \times 2}, \mathbf{0}_{2 \times 2}, \mathbf{C}_{3}\right]$, where $\mathbf{C}_{1}=\mathbf{C}_{2}=$ $\mathbf{C}_{3}=[1, \sqrt{0.8} ; \sqrt{0.8}, 1]$ and $\mathbf{C}_{1}=\mathbf{C}_{2}=\mathbf{C}_{3}=[1,0 ; 0,1]$ are for correlation and independence, respectively.

where $\mathbf{C}_{1}=\mathbf{C}_{2}=\mathbf{C}_{3}=[1, \sqrt{0.4} ; \sqrt{0.4}, 1]$. The lower and upper bounds converge to the asymptotic bound at high SNR.

Figure 4 shows a comparison of BER curves of SC for a 2-branch diversity system over correlated and independent Beaulieu-Xie fading channels with coherent BPSK modulation and a fading parameter of $m=1.5$ with a correlation matrix of the form $\mathbf{C}_{\mathbf{b}}=\left[\mathbf{C}_{1}, \mathbf{0}_{2 \times 2}, \mathbf{0}_{2 \times 2} ; \mathbf{0}_{2 \times 2}, \mathbf{C}_{2}, \mathbf{0}_{2 \times 2} ; \mathbf{0}_{2 \times 2}, \mathbf{0}_{2 \times 2}, \mathbf{C}_{3}\right]$, where $\mathbf{C}_{1}=\mathbf{C}_{2}=\mathbf{C}_{3}=[1, \sqrt{0.8} ; \sqrt{0.8}, 1]$ and $\mathbf{C}_{1}=$ $\mathbf{C}_{2}=\mathbf{C}_{3}=[1,0 ; 0,1]$ for correlation and independence, respectively. The performance improves when the channels are independent. The effect of the correlation matrix is also seen

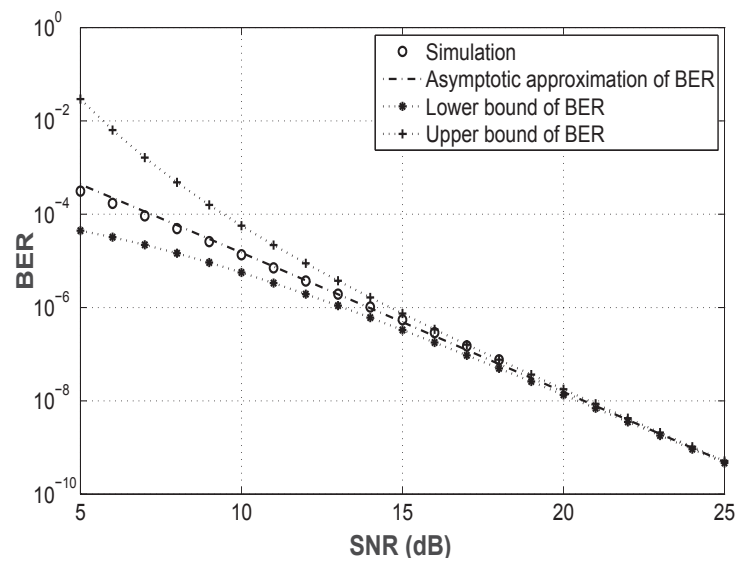

Fig. 3. BER curves for the asymptotic approximation, lower bound, and upper bound for SC, over correlated Beaulieu-Xie fading channels. The number of branches is $N=2$, the fading parameter is $m=1.5$, and the correlation structure is $\mathbf{C}_{\mathbf{b}}=\left[\mathbf{C}_{1}, \mathbf{0}_{2 \times 2}, \mathbf{0}_{2 \times 2} ; \mathbf{0}_{2 \times 2}, \mathbf{C}_{2}, \mathbf{0}_{2 \times 2} ; \mathbf{0}_{2 \times 2}, \mathbf{0}_{2 \times 2}, \mathbf{C}_{3}\right]$, where $\mathbf{C}_{1}=\mathbf{C}_{2}=\mathbf{C}_{3}=[1, \sqrt{0.4} ; \sqrt{0.4}, 1]$.

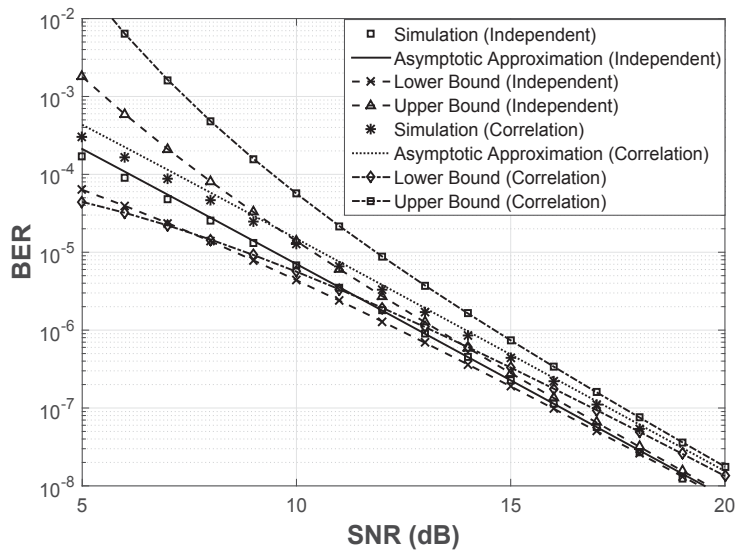

Fig. 4. BER curves for the asymptotic approximation, lower bound, and upper bound for MRC and EGC, over Beaulieu-Xie fading channels. The number of branches is $N=2$, the fading parameter is $m=1.5$, and the correlation structure is $\mathbf{C}_{\mathbf{b}}=\left[\mathbf{C}_{1}, \mathbf{0}_{2 \times 2}, \mathbf{0}_{2 \times 2} ; \mathbf{0}_{2 \times 2}, \mathbf{C}_{2}, \mathbf{0}_{2 \times 2} ; \mathbf{0}_{2 \times 2}, \mathbf{0}_{2 \times 2}, \mathbf{C}_{3}\right]$, where $\mathbf{C}_{1}=\mathbf{C}_{2}=\mathbf{C}_{3}=[1, \sqrt{0.8} ; \sqrt{0.8}, 1]$ and $\mathbf{C}_{1}=\mathbf{C}_{2}=\mathbf{C}_{3}=[1,0 ; 0,1]$ are for correlation and independence, respectively.

in the rate of convergence of the bounds, i.e., the higher the determinant of $\mathbf{C}_{\mathbf{b}}$ (signifying greater independence between channels), the faster the rate of convergence. Figure 5 shows a comparison between correlated and independent Beaulieu-Xie fading channels for a 3-branch diversity system. The rate of convergence in the 3-branch system is faster that of a 2-branch system.

\section{CONCLUSION}

In this work, we developed asymptotically tight lower and upper bounds for the outage probability and BER for the new Beaulieu-Xie fading model. The effect of the presence of more than one LOS component was seen (analytically) on the performance. It was ultimately found that this new fading 


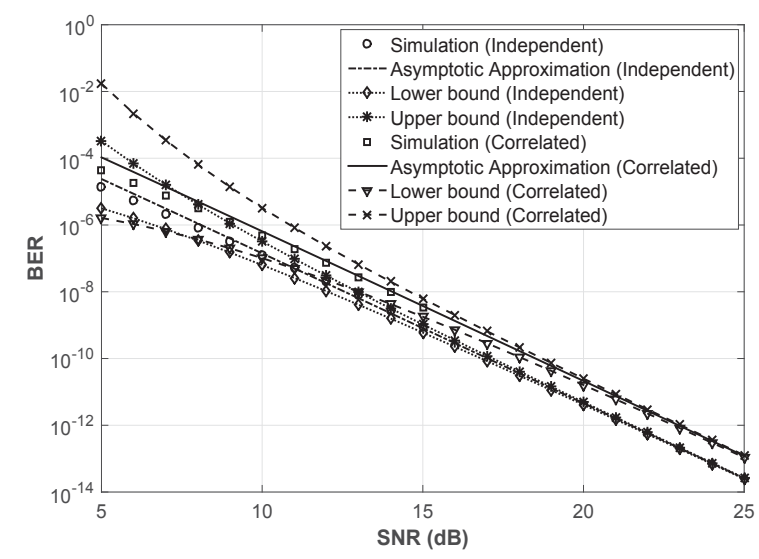

Fig. 5. BER curves for the asymptotic approximation, lower bound and upper bound for SC, over correlated and independent BeaulieuXie fading channels. The number of branches is $N=3$, the fading parameter is $m=1.5$, and the correlation structures is $\mathbf{C}_{\mathbf{b}}=$ $\left[\mathbf{C}_{\mathbf{1}}, \mathbf{0}_{3 \times 3}, \mathbf{0}_{3 \times 3} ; \mathbf{0}_{3 \times 3}, \mathbf{C}_{\mathbf{2}}, \mathbf{0}_{3 \times 3} ; \mathbf{0}_{3 \times 3}, \mathbf{0}_{3 \times 3}, \mathbf{C}_{\mathbf{3}}\right]$, where $\mathbf{C}_{1}=\mathbf{C}_{2}=$ $\mathbf{C}_{3}=[1, \sqrt{0.4}, \sqrt{0.2} ; \sqrt{0.4}, 1, \sqrt{0.4} ; \sqrt{0.2}, \sqrt{0.4}, 1]$ and $\mathbf{C}_{1}=\mathbf{C}_{2}=$ $\mathbf{C}_{3}=[1,0,0 ; 0,1,0 ; 0,0,1]$ are for correlation and independence, respectively.

model can be effective in characterizing wireless communication links with both LOS and NLOS components.

\section{REFERENCES}

[1] N. C. Beaulieu and J. Xie, "A novel fading model in channel with multiple dominant specular components," IEEE Wireless Commun. Lett., vol. 4, no. 1, pp. 54-57, Feb. 2015.

[2] M. D. Yacoub, "Nakagami-m phase-envelope joint distribution: A new model," IEEE Trans. Veh. Technol., vol. 59, no. 3, pp. 1552-1557, Mar 2010.

[3] N. C. Beaulieu and S. A. Saberali, "A generalized diffuse scatter plus line-of-sight fading channel model," in Proc. IEEE ICC 2014, Sydney, NSW, Jun., 2014, pp. 5849-5853.

[4] S. Wyne, A. P. Singh, F. Tufvesson, and A. F. Molisch, "A statistical model for indoor office wireless sensor channels," IEEE Trans. Wireless Commun., vol. 8, no. 8, pp. 4154-4164, Aug. 2009.

[5] M. Schwartz, W. R. Bennett, and S. Stein, Communication Systems and Techniques, New York: McGraw-Hill, 1996.

[6] V. V. Veeravalli, "On performance analysis for signaling on correlated fading channels," IEEE Trans. Commun., vol. 49, no. 11, pp. 1879-1883, Nov. 2001.

[7] V. A. Aalo, "Performance of maximal-ratio diversity systems in a correlated Nakagami-fading environment," IEEE Trans. Commun., vol. 43, no. 8, pp. 2360-2369, Aug. 1995.

[8] B. Zhu Fang Yang, J. Cheng, and L. Wu, "Performance bounds for diversity receptions over arbitrarily correlated Nakagami- $m$ fading channel," IEEE Trans. Wireless Commun., vol. 15, no. 1, pp. 699-713, Jan. 2016.

[9] R. A. Horn and C. R. Johnson, Matrix Analysis, 2nd ed. Cambridge, U.K.: Cambridge Univ. Press, 2013.

[10] E. W. Ng and M. Geller, "A table of integrals of the error function," Journal of Research of the National Bureau of Standards B, vol. 73, no. 1, pp. 1-20, Jan. 1969.

[11] S. Liu, J. Cheng, and N. C. Beaulieu, "Asymptotic error analysis of diversity schemes on arbitrarily correlated Rayleigh channel," IEEE Trans. Commun., vol. 58, no. 5, pp. 1351-1355, May 2010.

[12] X. Li and J. Cheng, "Asymptotic error rates analysis of selection combining on generalised correlated Nakagami- $m$ channels," IEEE Trans. Commun., vol. 60, no. 7, pp. 1765-1771, July 2012.

[13] S. Liu, J. Cheng, and N. C. Beaulieu, "Asymptotic error rates of EGC and SC on Rician channels with arbitrary correlation" in Canadian Workshop on Information Theory (CWIT), Ottawa, ON, May 2009, pp. 217-220.
[14] M. Z. Win, G. Chrisikos, and J. H. Winters, "MRC Performance for $M$ ary modulation in arbitrarily correlated Nakagami fading channels" IEEE Wireless Commun. Lett., vol. 4, no. 10, pp. 301-303, Oct. 2000.

[15] Q. T. Zhang, "Maximal-ratio combining over Nakagami fading channels with an arbitrary branch covariance matrix," IEEE Trans. Veh. Technol., vol. 48, no. 4, pp 1141-1150, July 1999.

[16] P. Lombardo, G. Fedele, and M. M. Rao, "MRC performance for binary signals in Nakagami fading with general branch correlation, " IEEE Trans. Commun., vol. 47, no. 1, pp. 44-52, Jan. 1999.

[17] I. S. Gradshteyn and I. M. Ryzhik, Table of Integrals, Series, and Products, 6th ed. New York, NY, USA: Academic, 2000. 